The authors present a patient suffering from malignant peritoneal mesothelioma. Differential diagnosis has become the major concern in the fatally ill patient. Pain, increasing abdominal girth, anorexia and weight loss, and recurrent ascites are the most frequent presenting symptoms. In this patient, fever of unknown origin was a clinical mask of mesothelioma. The diagnostic process was focused on infections and collagenvascular diseases since they are the most common causes of the systemic inflammatory response syndrome. However, persistent pyrexia can also occur, less frequently, in the course of any malignant disease.

Key words: malignant peritoneal mesothelioma, fever of unknown origin

\section{Fever of unknown origin: a clinical mask of malignant peritoneal mesothelioma}

\author{
Jacek Hermannn', Grzegorz Bajko², Marek Stajgis ${ }^{3}$, Jacek Szmeja ${ }^{1}$, \\ Tomasz Kościński ${ }^{1}$, Michał Drews ${ }^{1}$
}

1Department of General, Gastrointestinal and Endocrinological Surgery, Poznan University of Medical Sciences, Poznań, Poland

2Department of Internal Medicine, Private Clinic "Certus" in Poznań, Poland 3Department of Radiology, Poznan University of Medical Sciences, Poznań, Poland

\section{Introduction}

Mesothelioma is a primary neoplasm originating from the lining of serous cavities of the human body. It is an uncommon disease but with increasing prevalence. The pleural cavity is the most frequent localization of the disease, whereas approximately only one fifth of the lesions occur in the peritoneal cavity. Mesothelioma develops more commonly in males and the median age of diagnosis is 50 to 60 years [1]. There is a clear relationship between the neoplasm and asbestos exposure. Particularly, the peritoneal form of mesothelioma is associated with prolonged exposure to airborne asbestos fibres [2]. Mesothelioma occurs in the form of multifocal tumours and infiltrates of various size and shape affecting the parietal peritoneum and the visceral layer. Those lesions coalesce into larger nodules and tumours over time and may cover the entire peritoneal surface eventually [3]. Pain, increasing abdominal girth, anorexia and weight loss, and recurrent ascites are the most frequent presenting symptoms. In most cases, this insidious disease is recognized at an advanced stage under the clinical mask of carcinomatous peritonitis [4]. Most often, the final diagnosis is made after postoperative histopathological assessment supplemented with immunohistochemical tests. Malignant mesothelioma cells are characterized by positive staining for epithelial membrane antigen (EMA), Wilms tumour 1 (WT1), cytokeratins $(5 / 6,7)$ and calretinin, whereas there is absence of antigens such as the carcinoembryonic one [5]. The treatment of mesothelioma is palliative in most patients. Before 2000 , a combination of cytoreductive surgery and systemic chemotherapy was applied in most centres and the median survival rate ranged from 9 to 15 months. Afterwards, more aggressive methods of treatment were implemented, consisting of surgery, followed by intraperitoneal, hyperthermic, multidrug chemotherapy and whole abdominal radiation, and the rate of 5 -year survival increased to $50 \%$. However, such comprehensive management is undertaken only in patients after complete surgery, whereas the patients with incomplete cytoreduction, unresectable tumours like in the presented patient and those with extra abdominal lesions are candidates for palliative systemic chemotherapy [6, 7].

The article presents a 50-year-old male patient suffering from an incurable form of advanced malignant peritoneal mesothelioma under the clinical mask of fever of unknown origin.

\section{Case report}

The patient, R.O., age 50, tall and of heavy build, fell ill at the end of May 2009, being treated first in an out-patient department of internal medicine. He presented with symptoms of generalized viral infection such as fever, sore 
throat and myalgia, whereas physical examination was negative. Transient alleviation occurred after introduction of NSAIDs and clarithromycin, followed however by recurrent rises in temperature, paroxysmal and persistent cough, and malaise after a few days. Additional studies were negative except for an increase in erythrocyte sedimentation rate (ESR) to $95 \mathrm{~mm} / \mathrm{h}$, a rise in C reactive protein (CRP) to $46 \mathrm{mg} / \mathrm{l}$ and a single gallstone in the gallbladder found on ultrasonography. Cefuroxime, bronchodilatory, expectorant, and antitussic medications were administered with no improvement. AntiMycoplasma and anti-Epstein-Barr virus (EBV) antibody levels were assessed due to suspected atypical tracheitis. Whereas antibody titres were non-specific, there was still an increase in ESR to $120 \mathrm{~mm} / \mathrm{h}$. Because of persistent symptoms such as fever, weight loss (4 kg in a fortnight), hacking cough and rising ESR, the patient was suspected to have developed sarcoidosis. Then, he was admitted to the Private Clinic "Certus" on 23.06.2009. There were no abnormalities on physical examination. All the laboratory tests such as complete blood count (CBC), blood smear, liver and kidney function tests, cardiac enzyme studies, and tumour antigens were normal except for a further increase in CRP to $103.5 \mathrm{mg} / \mathrm{l}$ and non-specific changes on urinalysis. However, urine culture was negative. There were non-specific changes on the chest CT scan and the mediastinal lymph nodes were not enlarged. Amikacin, clindamycin and fluconazole were applied intravenously. Bronchofibroscopy was performed on 24.06.2009 and revealed signs of chronic, superficial bronchitis. The bacteriological tests such as Mycobacterium tuberculosis detection (MB/Bac T), cytological and mycological examination of the bronchial mucus secretion were negative. Due to persistent cough and recognized chronic bronchitis, the patient was given methylprednisolone orally, inhalatory steroids and bronchodilatory medications. Looking for other sources of infection, abdominal computed tomography (CT) was performed on 25.06.2009. This examination showed inflammatory infiltration along the wall of the hepatic flexure of the colon, a small amount of fluid under the right lobe of the liver, small lymph nodes in the liver hilus and along the aorta and a gallstone $4 \mathrm{~cm}$ in diameter in the gallbladder (Fig. 1). Normal echocardiography allowed endocarditis to be excluded. Metronidazole was ordered intravenously by the consultant surgeon, who suspected diverticulitis. Diverticula were located in the transverse, descending and sigmoid colon according to subsequent colonoscopy. The patient responded to the treatment, the temperature dropped and the cough went away. He was released home on 2.07.2009 in good general condition on intravenous amikacin for 5 days, to be followed by oral ciprofloxacin, ceftibuten, fluconazole, methylprednisolone and inhalatory ciclesonide and tiotropium. Due to recurrent fever up to $39^{\circ} \mathrm{C}$, malaise and further weight loss at the end of July he consulted a surgeon, who suspected the patient to have developed either perforation of the diverticulum or gallbladder empyema. He was admitted to the Department of Surgery on 31.07.2009. Pyrexia, malaise, mild abdominal pain in the right epigastrium and weight loss ca. $15 \mathrm{~kg}$ were the symptoms presented. Physical examination revealed an extensive, immovable mass with blurred borders in the right epigastrium with mild peritoneal signs. Laparotomy through the medi-
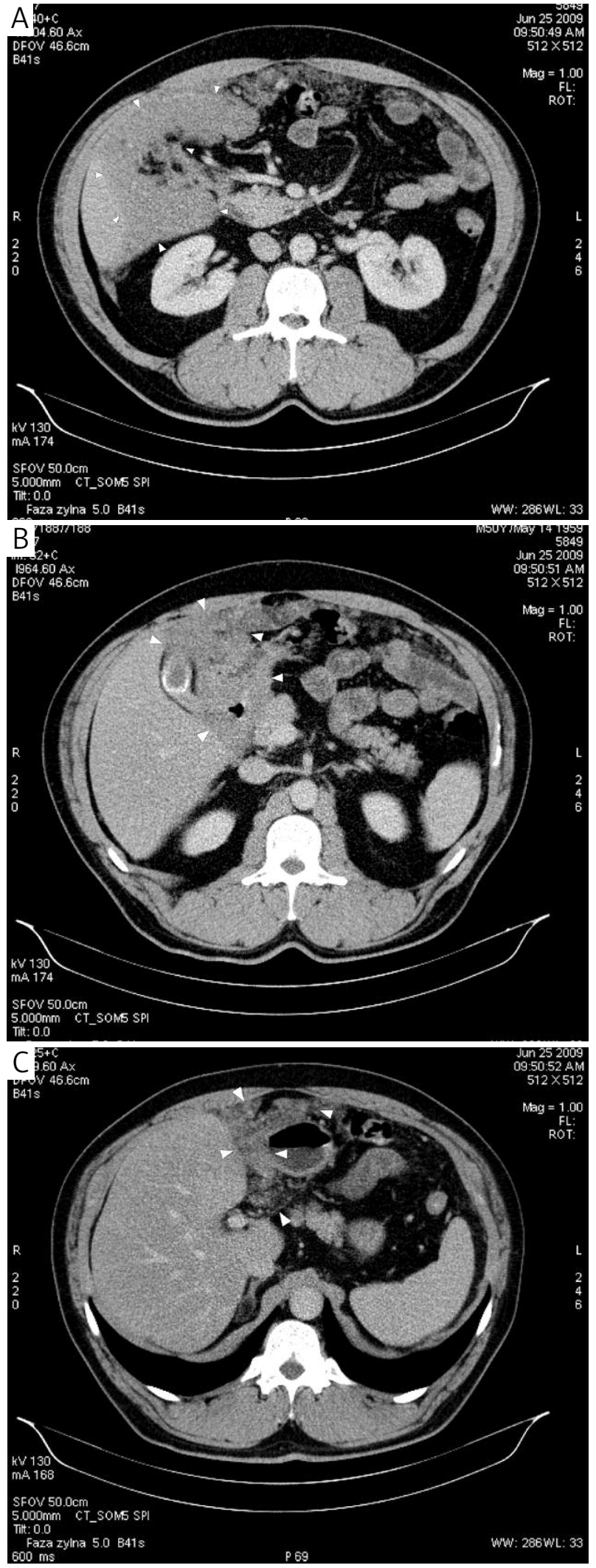

Fig. 1. Computed tomography, venous portal phase. Selected transverse sections at the level of the lower margin of the right lobe of the liver (A), liver hilus (B), and the antral part of the stomach (C). Extensive pathological mass infiltrating the visceral surface of the right liver lobe, the liver hilus, the hepatic flexure of the colon, the antral part of the stomach, and anterior wall of the abdomen in the middle and right epigastric region (white arrowheads) 
an incision was performed on 4.08.2009. There was a small amount of clear fluid in the peritoneal cavity, whereas the entire abdomen was filled with an unresectable omental mass which was encasing the colon, duodenum and small bowel loops, and reached the lateral walls of the peritoneal cavity and the minor pelvis. The parietal peritoneum was covered with multiple nodules similar to cancerous metastases from 1 to a few $\mathrm{mm}$ in diameter. There were enlarged lymph nodes in the liver hilus and in the vicinity of the stomach. There were no signs of gallbladder empyema, even though its wall was thick. Biopsy specimens were obtained from the omentum and peritoneal nodules and the abdomen was closed. The postoperative course was uneventful and the patient was released home on 12.08.2009. The postoperative histopathological assessment showed either low differentiated cancer or malignant mesothelioma. Negative immunohistochemical staining for CD20 excluded lymphatic origin of the neoplasm, whereas positive staining for WT1, cytokeratin 5/6 (CK5/6), CK7 and calretinin confirmed the diagnosis of the epithelial type of malignant mesothelioma. The patient was then admitted to the Department of Oncology on 2.09.2009 for chemotherapy. Despite intensive treatment his general condition deteriorated and he died on 14.09.2009. After death the patient's wife reported that he had lived in the vicinity of an asbestos factory until the age of 14 . The local road and his courtyard were hardened with asbestos.

\section{Discussion}

Differential diagnosis has become the major concern in the fatally ill patient. Pain, increasing abdominal girth, anorexia and weight loss, and recurrent ascites are the most frequent and non-specific presenting symptoms of peritoneal mesothelioma. Less common signs, such as persistent fever, night sweats [8] and mechanical bowel obstruction [9], are regarded as poor prognostic factors. Mesothelioma can be also associated with such paraneoplastic signs as thrombocytosis, hypoglycaemia, thrombophlebitis and liver failure [4]. Malignant peritoneal mesothelioma manifested clinically under the mask of fever of unknown origin in the presented 50-yearold male patient. Pyrexia of more than three weeks' duration, with temperatures over $38^{\circ} \mathrm{C}$ and a failure to identify the cause of the fever, is defined as fever of unknown origin [10]. Infections and collagen-vascular diseases are the most common causes of the systemic inflammatory response syndrome with elevated temperatures. The presented patient was suspected to have developed sarcoidosis due to the following symptoms: persistent fever, weight loss, non-productive cough and elevated ESR. Neither laboratory nor imaging studies confirmed the diagnosis. What is more, one evidenced source of infection was not identified [11]. However, persistent fever can also be a presenting sign of more frequently reported malignant diseases such as Hodgkin's lymphoma, leukaemia and renal cancer. Indeed, it has been observed that any other malignancy, either a localized tumour or already disseminated disease, can cause neoplastic fever. No clinical features reliably differentiate neoplastic fever from fever due to other causes. Therefore, neoplastic fever is a diagnosis of exclusion, confirmed after careful evaluation of other causes of pyrexia. Awaiting results of additional studies, an empirical treatment with standard broad-spectrum antibiotic therapy is followed for at least 7 days. If no clinical response to antibiotics occurs and there is no evident cause of infection, the naproxen test can be applied. Although the pathophysiology of neoplastic fever and its differences from other causes of fever remain uncertain, it has been shown that significantly more patients with neoplastic fever respond to naproxen compared to others. False positive results of the naproxen test are rare. Nevertheless, patients during naproxen treatment require further examination to rule out fever due to other nonneoplastic aetiology [12].

Computed tomography is the imaging method of choice for peritoneal mesothelioma. CT facilitates the diagnosis and evaluation of the clinical staging and resectability of the lesions, and it assists the percutaneous needle biopsy. Treatment is monitored with CT and it is also a prognostic factor of the neoplasm. A large tumour in the epigastric or hypogastric region, irregular or nodular peritoneal thickening limited to a single quadrant of the abdominal cavity and omental mass are the CT features of malignant mesothelioma. Additional findings that could be helpful in the differential diagnosis from other malignancies, including peritoneal carcinomatosis, are the lack of a primary lesion, encasement rather than infiltration of the adjacent organs, absence of lymph nodes or distant metastases and small amount of ascites [3]. A tumour exceeding $5 \mathrm{~cm}$ in diameter, nodular thickening of the peritoneum within the small bowel and its mesentery and segmental obstruction of the bowel indicate rather incomplete surgical debulking [13]. CT scans of the presented patient from 25.06.2009 were sent for reassessment to an independent radiologist who was not aware of the final diagnosis. He recognized an extensive either inflammatory or neoplastic mass ca. $16 \mathrm{~cm}$ in diameter, localized in the liver hilus and under the right lobe of the liver, spreading to the level of the lower pole of the right kidney and encasing the hepatic flexure of the colon, the antrum of the stomach and small bowel loops, infiltrating the anterior wall of the abdomen in the medial and right epigastric region (Fig. $1 \mathrm{~A}-\mathrm{C}$ ). A discrepancy between both $\mathrm{CT}$ results indicates difficulties in radiological evaluation of lesions originating from the peritoneum such as peritoneal studding, mesenteric thickening and omental mass.

However, magnetic resonance imaging (MRI), according to some authors, is superior to CT, both in the differentiation of malignant from benign lesions and because of better resolution in visualization of soft tissues. Perfusion and diffusion MRI are promising new techniques for the assessment of tumour cellularity and microvasculature and can be used for assessment of treatment response. In turn, positron emission tomography (PET) is also useful for the differentiation of benign from malignant lesions, and for staging, as well as monitoring response to treatment [14].

The authors declare no conflict of interest.

\section{References}

1. Bani-Hani KE, Gharaibeh KA. Malignant peritoneal mesothelioma. J Surg Oncol 2005; 91: 17-25.

2. Spirtas R, Heineman EF, Bernstein L. Malignant mesothelioma: attributable risk of asbestos exposure. Occup Environ Med 1994; 51: 804-11. 
3. Pickhardt PJ, Bhalla S. Primary neoplasms of peritoneal and sub-peritoneal origin: CT findings. RadioGraphics 2005; 25: 983-95.

4. Acherman YI, Welch LS, Bromley CM, Sugarbaker PH. Clinical presentation of peritoneal mesothelioma. Tumori 2003; 89: 269-73.

5. Ordonez NG. The diagnostic utility of immunohistochemistry and electron microscopy in distinguishing between peritoneal mesotheliomas and serous carcinomas: a comparative study. Med Pathol 2006; 19: 34-48.

6. Sugarbaker PH, Yan TD, Stuart OA, Yoo D. Comrehensive management of diffuse malignant peritoneal mesothelioma. Eur J Surg Oncol 2006; 32: 686-91.

7. Sas-Korczyńska B, Pęcak M, Korzeniowski S, Skołyszewski J. Wyni ki leczenia chorych na złośliwego międzybłoniaka opłucnej w materiale Centrum Onkologii w Krakowie. Wspołczesna Onkol 2005; 9: 386-92.

8. Chen L, Huang L, Wang J, Qian Y, Fang L. Malignant peritoneal mesothelioma presenting with persistent high fever. J Zheiiang Univ Sci 2011; 12: 381-4.

9. Sethna K, Sugarbaker PH. Localized visceral invasion of peritoneal mesothelioma causing intestinal obstruction: a new clinical presentation. Hepatogastroenterology 2005; 52: 1087-9.

10. Klastersky J, Weerts D, Hensgens C, Debusscher L. Fever of unexplained origin in patients with cancer. Eur J Cancer 1973; 9: 649-56.

11. Blackmon GM, Raghu G. Pulmonary sarcoidosis: a mimic of respiratory infection. Semin Respir Infect 1995; 10: 176-86.

12. Zell JA, Chang JC. Neoplastic fever: a neglected paraneoplastic syndrome. Support Care Cancer 2005; 13: 870-7.

13. Yan TD, Haveric N, Carmignani CP, Chang D, Sugarbaker PH. Abdominal computed tomography scans in the selection of patients with malignant peritoneal mesothelioma for comprehensive treatment with cytoreductive surgery and perioperative intraperitoneal chemotherapy. Cancer 2005; 103: 839-49.

14. Gill RR, Gerbaudo VH, Sugarbaker DJ, Hatabu H. Current trends in radiologic management of malignant pleural mesothelioma. Semin Thorac Cardiovasc Surg 2009; 21: 111-20.

\section{Address for correspondence}

\section{Jacek Hermann}

Department of General,

Gastrointestinal and Endocrinological Surgery

Poznan University of Medical Sciences

Przybyszewskiego 49

60-355 Poznań, Poland

phone +48618691275

$\mathrm{fax}+48618691684$

e-mail: jacekhermann@gmail.com

Submitted: $\quad 23.06 .2011$

Accepted: $\quad$ 18.01.2012 